\title{
Dénominations des maladies en langue populaire et argotique (de la 'synonymite' des noms de maladies)
}

\author{
Alicja Kacprzak \\ Université de Łódź, Pologne \\ Jean-Pierre Goudaillier \\ Université Paris Descartes, France
}

\begin{abstract}
Résumé : Pour ce qui est des diverses maladies pouvant être contractées par les êtres humains, on trouve de nombreuses appellations, aussi bien officielles que populaires et/ou argotiques.

L'analyse tant d'un point de vue formel que sémantique d'un certain nombre de ces désignations a été effectuée à partir d'un corpus constitué de relevés écrits (dictionnairiques et littéraires).

Il en apparaît une image de séries synonymiques de termes désignant certaines maladies. Cette "synonymite " de noms de maladies n'est pas certes gratuite : elle traduit un réel besoin langagier d'appeler le mal d'une manière spécifique selon l'usager et la situation de communication.

Mots-clés : terminologie médicale, variation diastratique, jargon, terme, synonymie.
\end{abstract}

Abstract: As far as illnesses which can be contracted by human beings are concerned one finds many designations, official or popular ones.

A formal as well as semantic analysis of a certain number of these names was carried out for the purpose of the present paper using a corpus established by written sources such as dictionaries and literary texts.

The result of this analysis consists of synonymous series of terms designating certain illnesses. This synonymy is not fortuitous. It shows a real linguistic need of calling a disease in a way particular to the speaker and the communication situation.

Keywords: medical terminology, diastratic variation, jargon, term, synonymy.

Parmi différentes langues spécialisées, la langue de la médecine se distingue, entre autres, par deux traits importants : son ancienneté et sa diversité sociolectale. En effet, il est sûr que la terminologie médicale se développe à travers les siècles au même rythme que la médecine. En même temps, appartenant aussi bien aux professionnels (médecins) qu'aux profanes (patients), elle présente un caractère soit scientifique soit quotidien, qui prévaut l'un sur l'autre, selon la situation de la communication. Ainsi, la variation diastratique de la langue médicale s'avère toute particulière et plusieurs niveaux de son lexique devraient être pris en considération :

-scientifique dans la communication officielle entre les médecins (lors des congrès, grandes conférences, etc.) ;

-scientifique dans la communication non-officielle entre les médecins (dans les salles d'hôpitaux, etc.) ;

- professionnel dans la communication entre le médecin et son patient; 
-non professionnel dans la communication entre les patients.

Si le premier niveau constitue un réservoir de termes spécialisés officiels, les trois autres, pour des raisons différentes, semblent favoriser la formation de termes non standard, voire populaires et argotiques, surtout en ce qui concerne les noms de maladies. Qui plus est, la différenciation diastratique du vocabulaire médical contribue aussi à son tour à l'apparition de dénominations nouvelles des pathologies. Et du point de vue diachronique, il s'avère que certains de ces noms succèdent aux appellations anciennes, tandis que d'autres ont été créés récemment. Vu cette complexité de sources lexicogéniques, il n'est point étonnant que le vocabulaire médical présente souvent une sorte de 'synonymite'l terminologique, état dans lequel plusieurs termes se rapportent au même phénomène. Dans cette situation, il nous semble intéressant de voir dans quelle mesure les appellations contemporaines, populaires et argotiques des maladies se situent dans une continuité par rapport à leurs anciennes dénominations.

Ayant adopté un point de vue sémasiologique, nous avons cherché dans un corpus d'ouvrages et de dictionnaires médicaux des termes se rapportant à quelques notions de notre choix. Leur analyse, tant historique que contemporaine, nous a permis de constater que différentes situations sont possibles.

1. Prenons le cas de l'entité nosologique définie dans le Dictionnaire des termes de médecine de Garnier et Delamare de 1989 comme: "inflammation aiguë du doigt ayant tendance à faire le tour de l'ongle ». Nommée pannarice dans La grande chirurgie de M. Guy de Chauliac en 1363, chez Boissier de Sauvages (1772) elle porte le nom de panaris, proche de celvi du terme scientifique actuele, à savoir panaris peri-unguéal. Quant au terme panaris, après extension de son sens, il constitue aujourd'hui dans le vocabulaire médical scientifique un nom générique donné à toutes les inflammations aiguës du doigt; à la fois, dans le langage standard il fonctionne toujours avec son ancien sens. Parmi ses deux termes synonymiques, tourniol et mal blanc, tous les deux notés par le TLFI sans aucun quantificateur, le premier seulement est attesté aussi par le Dictionnaire des termes médicaux de Garnier et Delamare (1989) ; par contre seulement le deuxième, mal blanc, apparaît sur les forums Internet consacrés à des maladies, ce qui pourrait confirmer sa valeur de terme populaire, ressenti comme un peu vieilli. Ainsi Coco51 écrit, en illustrant son texte de nombreuses émoticônes :

" j'ai un mal blanc aux dires des anciennes, mere et belle mere, et ça fait trop mal (e) ça m'a pris d'un coup vendredi soir mon doigt à triplé de volume au niveau de l'ongle et ça me lance, ça me reveille meme la nuit (-)

les mamies m'ont dit de plonger mon doigt dans l'eau de javel $\Theta$, mais je suis aller voir sur le net, et je flippe j'ai lu des trucs genre bacteries style staphilocoque doré $\Theta$, et ça parlait antibio voir intervention chirurgicale $\odot$ Quelqu'un a deja eu ça $\stackrel{\ominus}{\ominus}$ je vais prendre rdv demain chez le doc ... et plus loin : «merci Denisi, je reviens de chez le doc, alors c'est bien un mal blanc ou panaris $\%$.

2. Dans le cas de l'entité définie par Garnier et Delamare comme "tache de la cornée ॥, on note à travers les âges les deux dénominations taie et leucome. La

\footnotetext{
1 L'emploi du terme virtuel "synonymite", qui se rapporte à la surabondance de synonymes dans le langage médical, fait allusion au titre de l'article de Halina Lewicka, "Adiectivitis", publié dans Prace Filologiczne, XVIII, Warszawa, 1963, Mélanges W. Doroszewski, pp. 167-171.
} 
plus ancienne, taie, est attestée, selon le TLFI, déjà au Moyen Âge (dans Miserere de Renclus de Molliens); la deuxième, leucome apparaît dans les textes du XVIIIle siècle, notamment chez François Boissier de Sauvages qui explique: "leucome, en l'appelle en francois taie ", (1772, I tome: 369). Aujourd'hui les deux dénominations coexistent et sont notées aussi bien par le TLFI que par le Dictionnaire de Garnier et Delamare (1989). Cependant, le terme savant, leucome, semblant appartenir davantage à la variante scientifique du vocabulaire médical, démontre une fréquence inférieure sur les forums Internet à celle du terme taie, ressenti certainement à cause de sa forme comme plus populaire. La confirmation de ce fait se retrouve dans le dictionnaire Littré (en ligne) selon lequel taie est un «nom qu'on donne vulgairement aux diverses taches blanches et opaques qui se forment quelquefois sur la cornée ॥.

3. L'entité définie actuellement comme "tache cutanée rouge ou violacée, de taille variable, pâlissant à la pression ॥, a connu plusieurs appellations dès son apparition en nosologie. Boissier de Sauvages (1772) cite son nom populaire envie mais aussi son nom savant naevus. Si le premier existe toujours avec le statut de terme populaire, le deuxième fonctionne aujourd'hui comme un nom générique de "toute difformité cutanée d'origine embryonnaire", comme le note le dictionnaire spécialisé de Garnier et Delamare (1989). La médecine contemporaine recourt surtout aux dénominations volontairement opaques pour les non-initiés, que sont angiome plan et naevus flammeus. Le TLFI rappelle deux dénominations métaphoriques appartenant à la variante populaire du vocabulaire médical, tache de vin et fraise(s), que l'on retrouve dans le Garnier et Delamare.

4. Pour ce qui est du cancer, ce nom fonctionne en français contemporain comme synonyme de toute tumeur maligne. Ainsi par exemple le TLFI le définit comme "Tumeur maligne due à une multiplication anarchique des cellules d'un tissu organique ॥. Ce même terme en langue spécialisée ne s'applique qu'à des tumeurs qui se forment dans l'épithéliome, alors que ceux qui se développent dans d'autres tissus portent des noms différents, dont la motivation reste en liaison avec le caractère du tissu atteint, par exemple sarcome $<<$ gr. sarx 'chair'), ou avec la structure de la tumeur en question, par exemple myélome (< myélocyte). On retrouve l'une des premières attestations du terme cancer dans La grande chirurgie de M. Guy de Chauliac, (...) datant de 1363. Cet ouvrage, restitué par Laurens Jouber, a été publié à Tournon en 1598. Dans la table des matières, l'adresse Cancer renvoie à Chancre. II apparaît donc qu'à l'époque la distinction entre l'un et l'autre ne se faisait pas: "Chancre est équivoque à deux : sçavoir est à chancre qui est apostheme, duquel sera dict icy: \& à chancre qui est ulcère, duquel sera parlé cy dessouz. Chancre dont apostheme, est tumeur dure, ronde, veineuse, brune, croissante en peu de temps, ne donnant repos, chaud et douloureux " (1598: 144). Un peu plus loin l'auteur commente: "Les signes \& jugements sont, qu'il a substance dure, couleur livide \& obscure, veines à l'entour esluées, à la mode de pieds d'un chancre, avec douleur \& chaleur étrange. Le chancre de tout son genre est maladie pernicieuse (...), est une ladrerie particulière "), et rappelle une thèse bien connue d'Hippocrates de ne pas "toucher " aux tumeurs: "A quiconque les chancres sont occultes, il est meilleur de ne les curer pas, Car si on les cure, on en périt plutôt, si on ne les cure, on en dure plus long temps. ॥ (1598: 145). L'idée d'une maladie se propageant, rongeant l'organisme atteint se retrouve aussi chez Jean Auvray, dans "Le banquet des muses (...) ", publié en 1623, où l'auteur prévient cependant: "Si falloit-il ce chancre amputer de bonne heure, "l n'auroit pas gaigné les membres principaux. " Le nom de chancre apparaît 
aussi dans le Lexicon novum medicum graeco-latinum publié en 1690 par Steven Blankaart, qui à côté de chancre en français et krebs en allemand rappelle l'existence du terme grec carcinome et du mot latin cancer. Peu à peu, cette dénomination latine généralisera son emploi en français sous sa forme savante, en remplaçant la forme populaire, chancre; celle-ci se spécialisera à son tour pour désigner par la suite des ulcérations de différentes natures. Le XVIIIe siècle, époque de nomenclatures, cherchera à expliquer le nom de la maladie. Ainsi Noël Chomel dans son Dictionnaire oeconomique, (...) écrit: "Cette tumeur porte le nom du cancer par ressemblance à un Cancre de mer, ayant come lui plusieurs pieds et couleur obscure..." (1709: 6). De même Rober James dans le Dictionnaire universel de médecine, dans lequel l'entrée cancer renvoie à carcinoma, explique : "Les veines d'alenour sont gonflées et tendues, et forment à peu près la figure de pattes d'écrevisse ce qui leur a donné le nom de cancer qui signifie écrevisse en latin; d'autres veulent qu'on le nomme ainsi parce que semblable à cet animal qui serre fortement ce qu'il saisit et il est si opiniâtrement fixé sur la partie qu'il attaque qu'on ne l'en saurait retranché que très difficilement" (1746: 1622). À leur tour, Thomas Levacher de la Feutrie et François Moysant dans le Dictionnaire de chirurgie évoquent aussi l'identité entre le carcinome et le cancer et définissent le cancer comme "Tumeur dure, différemment figurée, inégale, douloureuse, livide ou plombée, environnée de plusieurs vaisseaux gonflés et variqueux qui ressemblent à peu près à des pattes d'écrevisse d'où cette tumeur a tiré son nom ॥ (1767: 252). La dénomination chancre revient seulement encore chez Hélian, qui dans son Dictionnaire du diagnostic, ou l'art de connoître les maladies, et de les distinguer exactement les unes des autres à l'entrée Chancre (distincte de l'entrée Chancre vénérien) indique: "voyez Cancer" (1771: 46). À une année près, François Boissier de Sauvages place le terme cancer qu'il donne comme synonyme de Carcinome, mais aussi de Tumeur Chancreuse et de Cancer occulte, dans la Classe des Vices et dans l'Ordre des Phyma, en expliquant en même temps que le «bas peuple ne le nomme point, ou (...) le nomme simplement le Méchant ", car selon la croyance populaire "ce mal s'aigrit comme le charbon, lorsqu'on le nomme par son nom " (1772, $I X$ : 528). Les $X I X X^{e}$ et $X X X^{e}$ siècles gardent pourtant la forme cancer qui acquiert même parfois dans la langue des professionnels la valeur d'un hyperonyme, comme en témoigne cette définition donnée par le Dictionnaire des termes de médicine de Garnier et Delamare : "Nom donné à toutes les tumeurs malignes qui s'étendent rapidement et ont tendance à se généraliser ». Quelques lignes plus loin on précise quand même: "(...) ce mot s'applique surtout aux épithéliomes ॥ (1989: 133). La fréquence de la maladie, ainsi que son caractère quasi irrévocable sont certainement encore actuellement à la base de la recherche de l'euphémisme. Ainsi, les sites Internet consacrés à la santé énumèrent d'autres appellations du cancer, notamment néoplasme, néoplasie, néoformation et tumeur qui peuvent aussi bien se rapporter à des formations bénignes, mais souvent utilisées pour éviter le nom de cancer, dont le poids émotionnel est particulièrement lourd. D'autres motifs semblent avoir provoqué l'apparition relativement récente de la dénomination crabe. S'agirait-il d'un augmentatif expressif, lorsqu'un internaute s'écrie "Je hais le crabe qui l'a emporté ! ॥ à la nouvelle de la mort des suites d'un cancer fulgurant de Fred Chichin, guitariste d'un groupe de rock. II est frappant en tout cas qu'à côté des synonymes expressifs on note une quasi absence de synonymes populaires, voire argotiques de cancer, malgré la (mauvaise) notoriété de la maladie.

5. Les appellations argotiques, quant à elles, semblent être plus nombreuses, lorsque l'entité nosologique en question appartient surtout aux milieux de la 
pègre, de la prostitution, ce qui est le cas des maladies vénériennes. Non que ce type de pathologies épargne les personnes des milieux favorisés, mais parce que certainement il y a été tabou, ce qui n'a pas été le cas du milieu des maisons publiques, etc. Le cas intéressant est constitué par la maladie définie comme: "maladie infectieuse dont l'agent pathogène est le gonocoque " (Garnier et Delamare, 1989: 113). Connue sous plusieurs appellations, parmi lesquelles le plus souvent appliquées celles de chaude-pisse ou chaudepisse, avec l'orthographe variable, elle est citée entre autres dans le Traité d'économie rurale composé en Angleterre du XIIIème, comme l'atteste le TLFI, sous le nom de chaude-pisse. On retrouve ensuite ce terme chez Ambroise Paré, par lequel il est d'ailleurs utilisé en 1564 au pluriel avec la valeur du singulier, comme dans le titre même du chapitre: "Des chaudes-pisses et carnositez engendres au meat urinal $॥$. Paré y propose une distinction entre la chaude-pisse (qui est synonymique avec ardeur d'urine) et la gonorrhée, ce qui prouve que les deux termes fonctionnent déjà à cette époque-là : « Aucuns ont iusques icy pensé que la Chaude-pisse eust quelque chose de commun avec la Gonorrhee des anciens: mais elles sont fort differentes l'une de l'autre(...). Car la gonorrhee est un flux de semence involuntaire, decoulant de toutes les parties de corps aux parties genitalles (...). Au contraire, la Chaude-pisse, ou ardeur d'urine, est une sanie qui sort par la verge (...) avec douleur " (1564: 132). Plus de cent ans après, Charles de Barbeyrac intitule un des chapitres de son Traitez nouveaux de médecine (1684) «De la gonorée virulente ou de la chaude-pisse ", ce qui montre que les deux termes sont à l'époque utilisés comme synonymes. Cependant Chomel tout au début du XVIIIe siècle ne retient que le terme de gonorée, en indiquant que pour la soigner « de trois en trois jours il faut faire saigner le Malade " (1709: 11). Pour Boissier de Sauvages, gonorrhée constitue un Genre appartenant à l'Ordre des Flux séreux, et se divise en plusieurs espèces: gonorrhée simple, gonrrhoea libidinosa, gonrrhoea oneirogonos et gonorrhoea syphilitica; cette dernière, appelée aussi chaude pisse ou gonorrhée virulente, "est celle qu'on contracte par un commerce impur " (1772). Dans l'encyclopédie de Panchoucke, on mentionne deux termes, gonorrhée et chaudepisse virulente, en insistant sur le fait que "ce sont cependant deux maladies différentes" (1792: 669). L'une des éditions postérieures de ce même ouvrage évoque pour la première fois encore une autre appellation du mal en question : «Le docteur Swediaur a substitué au mot gonorrhée celui de blennoragie " (1817:4). À son tour, André Lebel dans son Vade-mecum (...) écrit : "La blennorragie ou gonorrhée a reçu encore bien d'autres noms, tels que ceux d'urétrite virulente, vénérique, syphilique, écoulement, chaudepisse ou échauffement " (1858:28). Ce dernier terme, échauffement, relativement rare dans les sources analysées, est noté par le TLFI comme 'vieilli' ou 'populaire', avec l'explication qu'il s'agit d'un "Malaise général ou localisé se manifestant par un excès de chaleur, de l'inflammation, des démangeaisons ॥. Notons aussi le terme très imagée de chaude-lance, évoqué notamment dans la Double chance d'Arnoux: «Moi (...) confident des chaudes-lances, des bistrots et des ménopauses des épicières (1958: 28). Céline en fait un autre emploi dans la Mort à crédit: "lls sont toujours pressés des genres de malades, ils ont peur que ça revienne plus la bandaison des familles. La mère Vitruve en venant me voir elle avait repéré ces choses-là... Les petits jeunes hommes à la "chaude-lance» leur première, ça les rend tout mélancoliques, ça les affecte énormément. Elle venait attendre à la sortie... Elle leur faisait au sentiment... à la touchante sollicitude... " Ça te cuit fort hein, mon petit gars ?... Je sais ce que c'est... J'en ai soigné... " (1936: 526). À la page suivante, pour parler de la même maladie, Céline cite encore un terme 
argotique, chtoville: "Voici le "dossier 34 ", l'employé aux lorgnons noirs, le timide, le petit futé, il va l'attraper sa chtouille tout exprès, chaque six mois, cour d'Amsterdam, pour mieux expier par la verge... il pisse ses lames de rasoir dans les connasses des petites annonces... ॥ (1936:527-528).

Les analyses effectuées afin de voir dans quelle mesure les appellations contemporaines des maladies continuent leurs anciennes dénominations et quel est le rapport entre les appellations fonctionnant aux siècles passés et celles d'aujourd'hui, populaires et argotiques, nous ont permis d'observer que plusieurs cas de figures sont possibles, à savoir :

- le nom populaire actuel continue le nom standard ancien (tel est le cas par exemple du terme chaude-pisse, mais aussi de pierre) ;

- le nom populaire actuel prolonge le nom populaire ancien (c'est le cas du terme envie) ;

- le nom populaire actuel est original par rapport aux appellations anciennes (tels sont les cas de tache de vin, chaude-lance, crabe, mais aussi de caillou).

II est donc bien visible qu'il n'y a aucune régularité dans le fonctionnement des termes médicaux non standard qui peuvent aussi bien témoigner d'une longue existence dans la variante officielle ou non officielle de la langue de la médecine qu'être apparus il y a relativement peu de temps.

Il est sûr par contre que les noms populaires de maladie présentent une prédilection pour certains types lexicogéniques. Leur tropoïsation constitue sans doute un signe important de leur expressivité. En effet, dans la majorité des cas, ces dénominations ont un caractère imagé de métaphores (c'est notamment le cas de tels noms que crabe ou tache de vin) ou de métonymies (comme dans le cas des termes envie et chaude-pisse).

Il convient aussi de souligner une richesse synonymique, tant chronologique que diastratique, qu'entraînent certaines notions. Pour ce qui est de l'histoire des dénominations médicales, leurs changements ont souvent été liés au développement du savoir et de la science dans ce domaine. En effet, la cristallisation de notions a plus d'une fois donné lieu à une modification terminologique. En même temps, pour des raisons variées, externes et internes à la langue, les termes spécialisés changent parfois de registre, évoluant librement entre le standard et le non standard, l'officiel et le non officiel. Sans pouvoir définir clairement et strictement ces raisons, il est possible cependant de constater quelles sont les fonctions de cette 'synonymite' dans les dénominations populaires de certaines maladies. II semble d'une manière générale que ces noms imagés puissent servir à rendre familier le mal, à l'apprivoiser, à le dominer de cette façon dans la mesure du possible. Ceci, par exemple en expliquant son origine. Le mot envie désignant l'angiome plan explique que l'affection est due à une envie d'un aliment qu'avait la mère de l'enfant pendant la grossesse, d'où vient à la fois une prescription aux femmes enceintes de ne pas exprimer d'envies pour éviter une telle stigmatisation à leurs enfants. On peut dompter le mal aussi en évoquant son aspect d'une façon plus directe. Ainsi l'appellation mal blanc s'oppose-t-elle par son caractère évident à panaris, plus obscure. La maladie semble moins grave aussi quand elle est minimisée, ne serait-ce que par l'emploi d'un nom ayant une bonne connotation. Tel semble être le cas de l'appellation caillou qui remplace parfois 
calcul; l'aspect anodin du mot évoquant la nature et même les vacances éloigne d'une certaine façon tout ce qui est lié à calcul : maladie, douleur, souffrance. Or, il est possible aussi de dominer le mal en le dévaluant. C'est le cas de dénominations telles que crabe pour cancer et chaude-lance pour blennorragie. Dans le premier cas, l'emploi du nom d'un crustacé pour appeler une maladie grave lui enlève sans doute de son caractère redoutable, la ridiculise même. Dans le deuxième cas, l'appellation presque vulgaire, chaude-lance, permet de traiter le mal avec plus de distance, de diminuer peut-être même son importance. Notons enfin le cas de la dénomination populaire méchant, utilisé au XVIIIe siècle pour éviter de nommer directement le cancer: ainsi en ôtant son nom, on cherchait à rendre le mal inopérant.

\section{Bibliographie}

AUVRAY, J. (1636) : Le Banquet des muses, ou les Divers satires. Rouen : D. Ferrand.

BARBEYRAC, Ch. de (1684): Traités nouveaux de médecine, contenans les maladies de la poitrine, les maladies des femmes, \& quelques autres maladies particulières: selon les nouvelles opinions. Lyon : Jean Certe.

BLANKAART, S. (1690): Lexicon novum medicum graeco-latinum. Lugduni Batavorum : Apud Cornelium Boutesteyn - Jordaanum Luchtmans.

BOISSIER DE SAUVAGES DE LACROIX, F. (1772) : Nosologie méthodique de distribution des maladies en classes, en genres et en espèces suivant l'esprit de Sydenham et la méthode des botanistes. Lyon : Jean-Marie Bruyset, Imprimeur-Libraire.

BOUCHE, P. (1994) : Les mots de la médecine. Paris : Belin.

CÉLINE, L-F. (1936) : Mort à crédit. Paris : Gallimard.

CHAULIAC, G. de (1598) : La grande chirurgie de M. Guy de Chauliac, 1363, (...), restituée par M. Laurens Joubert. Tournon : Claude Michel.

CHOMEL, N. (1709): Dictionnaire œconomique, contenant divers moyens d'augmenter et conserver son bien, et même sa santé ... Avec plusieurs remedes ... pour un très grand nombre de maladies (...). Paris: Le Conte \& Montalant.

GARNIER, M. et J. DELAMARE (1989): Dictionnaire des termes de médecine. Paris: Maloine.

GOUDAILLIER, J.-P. (2001): «De l'art de susciter la panique par l'appellation des maladies en langue populaire et argotique ॥. Colloque Les mots de la santé (3) - Mots de la santé et psychoses, Université Lyon 2, 11-12 décembre 2008. In Travaux du CRIT, Les mots de la santé (3) - Mots de la santé et psychoses. Paris: L'Harmattan, pp. 31-38.

- (2001 [1997]) : Comment tu tchatches !-Dictionnaire du français contemporain des cités. Paris : Maisonneuve \& Larose.

GUILLEMEAU, J. (1621): De la Grossesse et accouchement des femmes ; du gouvernement de celles-ci et moyen de survenir aux accidents qui leur arrivent, ensemble de la nourriture des enfans, par feu Jacques Guillemeau,... augmenté de... plusieurs maladies secrettes, avec un traité de l'impuissance, par Charles Guillemeau. Paris : Abraham Pacard. 
HÉLIAN, M. (1771) : Dictionnaire du diagnostic, ou l'art de connoître les maladies, et de les distinguer exactement les unes des autres. Paris : Vincent.

JAMES, R. (1746) : Dictionnaire universel de medecine. Paris : Briasson - David l'aîné Durand.

KACPRZAK, A. (à paraître): "Psychose et propagande: épidémie de variole, Wroclaw $1963 \%$. Colloque Les mots de la santé (3) - Mots de la santé et psychoses, Université Lyon 2, 11-12 décembre 2008.

- (2000): Terminologie médicale française et polonaise - analyse formelle et sémantique. Łódź : Wydawnictwo Uniwersytetu Łódzkiego.

- (2009) : "La métaphore dans le jargon médical». A. KACPRZAK et J.-P. GOUDAILLIER (éds.) : Standard et périphéries de la langue. Łódź : LEKSEM, pp. 109-116.

LEBEL, A. (1858) : Vade-mecum des jeunes gens, ou Guide pratique des maladies syphilitiques... précédé d'un essai sur l'onanisme. Paris : J.-B. Baillière et fils.

LEVACHER DE LA FEUTRIE, T., F. MOYSANT et E. DE LA MARCELLERIE (1767): Dictionnaire de Chirurgie. Paris : Lacombe.

PARÉ, A. (1564): Dix livres de la chirurgie : avec le magasin des instrumens necessaires à icelle. Paris : Jean Le Royer.

SOURNIA, J.-Ch. (1997) : Histoire de la médecine. Paris : La Découverte - Poche.

\section{Sitographie}

FRANTEXT : PATIN, G. (1649) : Lettres.

FRANTEXT : SERRES, O. de (1603) : Le Théâtre d'agriculture et mesnage des champs. <http://www.e-sante.be/>

<http://www.vulgaris-medical.com/encyclopedie-medicale> 